\title{
DAPAGLIFLOZIN CLINICAL EXPERIENCE AND SAFETY ON PATIENTS WITH TYPE 2 DIABETES AND OBESITY
}

MM Roca-Rodríguez (1), MJ Picón-César (2), AJ Omiste-Romero (2), J García-Pérez (1), M Aguilar-Diosdado (3).

ECE2016

28 - 31 May

Munich

Germany
1 Department of Endocrinology, La Linea de la Concepcion Hospital, Cadiz, Spain. 2 Department of Endocrinology, Virgen de la Victoria and Regional Hospitals, Malaga, Spain.

3 Department of Endocrinology, Puerta del Mar Hospital, Cadiz, Spain.

\section{INTRODUCTION}

New treatments of diabetes, such as Dapagliflozin, improve global metabolic status beyond glycemic control.

\section{AIM}

To evaluate tolerance to Dapagliflozin and its effects on metabolic control in type 2 diabetes and obese patients attended in Endocrinology offices.

\section{METHOD}

A prospective study of patients with type 2 diabetes and obesity. In an intra-subject analysis, clinical and analytical data were evaluated at baseline and after Dapagliflozin treatment.

\section{RESULTS}

We studied 38 patients (47.4\% women) with type 2 diabetes and obesity. Average age was $55.7 \pm 1.5$ years, average duration of diabetes was $10.7 \pm 1.7$ years and $40.9 \%$ and $91.3 \%$ had family history of cardiovascular disease and diabetes, respectively. At baseline, $78.9 \%$ of the patients had metformin, $39.5 \%$ others oral hypoglycemic agents, $13.2 \%$ GLP-1 agonists and $44.7 \%$ insulin. We re-evaluated the patients $3.9 \pm 0.2$ months after treatment with Dapagliflozin.

No changes in levels of creatinine and glomerular filtration rate were observed.

Regarding tolerance to Dapagliflozin, only $7.9 \%$ developed urinary tract infections.
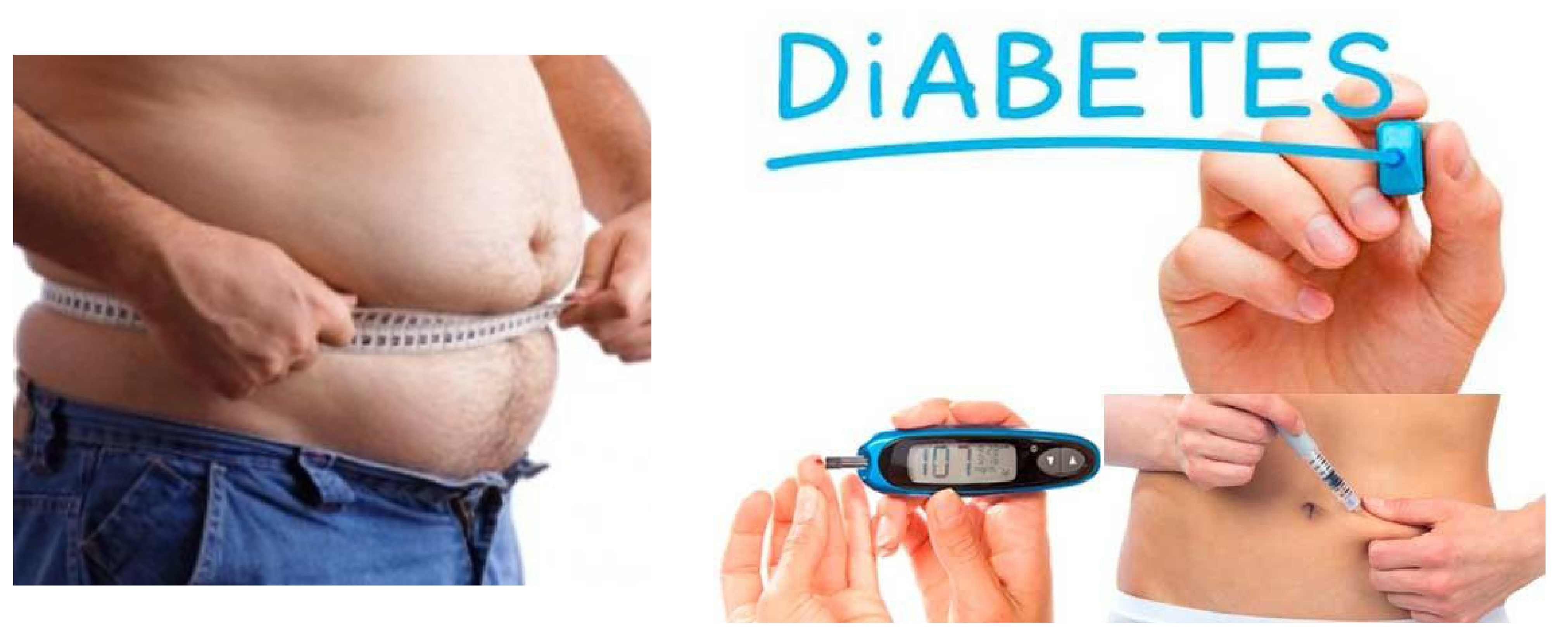

\section{CONCLUSIONS}

1) Significant improvement of anthropometric parameters and glycemic control in terms of fasting glucose and $\mathrm{HbA} 1 \mathrm{c}$, and significant improvements of blood pressure, GPT and lipid profile were observed.

2) Only $7.9 \%$ developed urinary tract infections.

3) In addition, we found a significant intensification of lipid-lowering therapy.

Table 1: Clinical and laboratory variables.

\begin{tabular}{|c|c|c|c|}
\hline Variable & Basal & Dapagliflozin & $P$ \\
\hline Weight $(\mathrm{kg})$ & $96.8 \pm 2.4$ & $92.8 \pm 2.4$ & $<0.001^{*}$ \\
\hline BMI (kg/m2) & $35 \pm 0.9$ & $33.4 \pm 0.8$ & $<0.001^{*}$ \\
\hline $\mathrm{SBP}(\mathrm{mmHg})$ & $146.9 \pm 3.6$ & $136.5 \pm 3.2$ & $0.008^{*}$ \\
\hline $\mathrm{DBP}(\mathrm{mmHg})$ & $83.4 \pm 1.8$ & $79.4 \pm 1.8$ & $0.030^{*}$ \\
\hline HR (bpm) & $82 \pm 1.9$ & $79.9 \pm 1.8$ & 0.730 \\
\hline $\begin{array}{l}\text { Fasting glucose } \\
\text { (mg/dL) }\end{array}$ & $188 \pm 13.9$ & $141.3 \pm 6.7$ & $0.001^{*}$ \\
\hline $\mathrm{HbA} 1 \mathrm{c}(\%)$ & $8.6 \pm 0.3$ & $7.4 \pm 0.2$ & $<0.001^{*}$ \\
\hline Total-Chol (mg/dL) & $186.1 \pm 7.8$ & $170 \pm 6.6$ & $0.011^{*}$ \\
\hline LDL-Chol (mg/dL) & $107.8 \pm 7.3$ & $94.4 \pm 5.1$ & $0.038^{*}$ \\
\hline HDL-Chol (mg/dL) & $41.3 \pm 1.5$ & $45.6 \pm 2$ & 0.145 \\
\hline $\mathrm{TG}(\mathrm{mg} / \mathrm{dL})$ & $228.6 \pm 27.4$ & $174.5 \pm 13.3$ & $0.025^{*}$ \\
\hline GOT (UI/L) & $29.4 \pm 6.8$ & $22.6 \pm 1.9$ & 0.408 \\
\hline GPT (UI/L) & $44.2 \pm 10$ & $31.5 \pm 3.8$ & $0.032^{*}$ \\
\hline GGT (UI/L) & $48.5 \pm 10$ & $42.7 \pm 15.2$ & 0.135 \\
\hline Creatinine & $0.7 \pm 0.1$ & $0.8 \pm 0.1$ & 0.336 \\
\hline $\begin{array}{l}\text { Glomerular } \\
\text { filtration rate }\end{array}$ & $90.1 \pm 2.7$ & $84.9 \pm 3.9$ & 0.188 \\
\hline $\begin{array}{l}\text { Albumin/creatinine } \\
\text { ratio }(\mathrm{mg} / \mathrm{L})\end{array}$ & $11.9 \pm 3.2$ & $51.9 \pm 33.9$ & 0.188 \\
\hline $\begin{array}{l}\text { Albumin/creatinine } \\
\text { ratio }(\mathrm{mg} / \mathrm{g})\end{array}$ & $187.3 \pm 157.2$ & $194.7 \pm 137.7$ & 0.364 \\
\hline Insulin units & $34.3 \pm 7.6$ & $30.4 \pm 6.9$ & 0.390 \\
\hline $\begin{array}{l}\text { Antihypertensive } \\
\text { drugs (\%) }\end{array}$ & 63.2 & 63.9 & 0,500 \\
\hline Lipids drugs (\%) & 57.9 & 75 & $<0.001^{*}$ \\
\hline
\end{tabular}

